\title{
Modelling the Anti-Migraine Effects of BIBN 4096 BS
}

\section{A New Calcitonin Gene-Related Peptide Receptor Antagonist}

\section{Iñaki F. Trocóniz, ${ }^{1}$ Jan-Markus Wolters, ${ }^{2}$ Christiane Tillmann, ${ }^{3}$ Hans G. Schaefer ${ }^{3}$ and} Willy Roth ${ }^{3}$

1 Department of Pharmacy and Pharmaceutical Technology, School of Pharmacy, University of Navarra, Pamplona, Spain

2 Department of Project Management R\&D, Boehringer Ingelheim Pharma GmbH \& Co. KG, Biberach, Germany

3 Department of Drug Metabolism and Pharmacokinetics, Boehringer Ingelheim Pharma GmbH \& Co. KG, Biberach, Germany

\section{Abstract}

Background and objective: Migraine attacks are associated with release of the calcitonin gene-related peptide (CGRP) from trigeminal nerves. BIBN 4096 BS is the first CGRP receptor antagonist tested in humans showing response rates similar to those reported for triptans, together with very good safety and tolerability profiles. The objective of the current study is to develop a population pharmacokinetic/pharmacodynamic model resembling the mechanism of action of BIBN 4096 BS, and to extract by model-based simulations dosage formulations and pharmacodynamic properties that can assist in the development of CGRP receptor antagonists.

Methods: 126 patients with an acute moderate to severe migraine attack lasting not more than 6 hours were enrolled in this phase IIa study. BIBN 4096 BS was given as a single intravenous 10-minute infusion at different dose levels ranging from 0.25 to $10 \mathrm{mg}$. Severity of headache was measured up to 24 hours. Patients who did not show pain relief by 2 hours were allowed to take rescue medication. Severity of headache and time to rescue medication measurements were fitted simultaneously using logistic regression and time-to-event analysis with nonlinear mixed-effect modelling software NONMEM version $\mathrm{V}$.

Results: Severity of headache and time to rescue medication were described as a function of the fraction of the CGRP receptors blocked by BIBN 4096 BS, and controlled by the second- and first-order rate constants representing the onset $\left(\mathrm{k}_{\text {on }}\right)$ and offset $\left(\mathrm{k}_{\text {off }}\right)$ of the anti-migraine effects. The model predicted a slow rate of offset of the anti-migraine effect (half-life of $\mathrm{k}_{\mathrm{off}}=21$ hours). The model developed described the data well and was validated properly.

Discussion: A semi-mechanistic population pharmacokinetic/pharmacodynamic model has been developed for the anti-migraine effects of BIBN 4096 BS, characterised by the severity of headache and time to rescue medication. Simulations exploring the effect of the rate of absorption, bioavailability after an 
extravascular administration and the rate of activation/inactivation of the anti-migraine effect were performed. The rate of absorption seems to play a minor role; however, at least bioavailability fractions of $0.2-0.3$ should be obtained. With regard to the kinetics of the anti-migraine effect, and to achieve a response rate of $60 \%$ at 2 hours, values of $\mathrm{k}_{\text {on }}$ should be $>0.081 \mathrm{~mL} / \mathrm{ng} / \mathrm{h}$. At later times after administration higher values of $\mathrm{k}_{\text {off }}$ are associated with faster offset of the response. The simulations showed that molecules with high $\mathrm{k}_{\text {on }}$ and low $\mathrm{k}_{\text {off }}$ values are the most promising.

\section{Background}

Migraine is a very incapacitating transient disease affecting $12 \%$ of the general population, with a pathogenesis that is still not completely understood. ${ }^{[1]}$ Nevertheless it is known that the release of the calcitonin gene-related peptide (CGRP) from trigeminal nerves is associated with migraine attacks. ${ }^{[2]}$ CGRP is a potent dilator of brain and dura vessels and therefore it plays an important role in regulating blood flow to the brain and pain-sensitive meninges, and can also cause degranulation and subsequent release of inflammatory agents from meningeal mast cells. ${ }^{[3,4]}$

A decrease in CGRP levels at the brain area can be elicited by several mechanisms, for example the inhibition of the release of CGRP. This mechanism of action is used by the triptans, which have affinity for multiple serotonin receptors, thereby producing vasoconstriction of cerebral vessels, but also of coronary vessels. ${ }^{[5,6]}$ Another alternative is to block the binding between CGRP and its receptor, for which an antagonist of the CGRP receptor has to be availa-

Table I. Summary of patient characteristics and migraine history

\begin{tabular}{ll}
\hline Characteristic & Value $^{\mathrm{a}}$ \\
\hline No. of patients (male/female) & $126(26 / 100)$ \\
Age $(\mathrm{y})$ & $45.5(21.0-54.0)$ \\
Weight $(\mathrm{kg})^{\mathrm{b}}$ & $69.5(48.0-119)$ \\
Height $(\mathrm{cm})^{\mathrm{b}}$ & $169(150-190)$ \\
Time since first diagnosis (y) & $22.2(0.9-53.3)$ \\
Number of attacks in the last 6 months & $19.6(5-72)$ \\
Patients with history of attacks with aura & $37(29.4)$ \\
[n (\%)] & \\
\hline a Values are expressed as mean (range), unless otherwise \\
b $\quad$ Weight and height are missing for one patient. \\
\hline
\end{tabular}

ble. BIBN $4096 \mathrm{BS}$ is a non-peptide CGRP receptor antagonist that based on in vitro and in vivo preclinical studies shows very high affinity and specificity for the human CGRP receptor. ${ }^{[7,8]}$

Only very recently clinical data have become available. In a proof of concept study where BIBN 4096 BS was administered intravenously by a 10 minute infusion to patients with migraine attacks rated as moderate to severe, the investigators found response rates similar to the efficacy rates reported for triptans, together with good safety and tolerability profiles. ${ }^{[9]}$ In addition, the pharmacokinetics of BIBN 4096 BS have been characterised by means of non-compartmental and population pharmacokinetic analyses. BIBN 4096 BS showed a linear predictable pharmacokinetic behaviour that was not mainly affected by standard demographic characteristics. $^{[10,11]}$

Based on the pharmacokinetic and pharmacological profiles of BIBN 4096 BS, sufficiently high rate both of absorption and bioavailability after extravascular administration will be crucial for achieving therapeutic plasma concentrations. To have a tool allowing exploring in silico an important clinical end-point (i.e. response rate at 2 hours after drug administration) based on different absorption characteristics and/or receptor binding properties would be very useful. Therefore the aim of the current manuscript is to develop a population pharmacokinetic/pharmacodynamic model resembling as much as possible the mechanism of action of BIBN 4096 BS, and to extract by model-based simulations dosage formulations and pharmacodynamic properties that can assist in the development of compounds from the new class of CGRP receptor antagonists. This is particularly challenging in the present work 


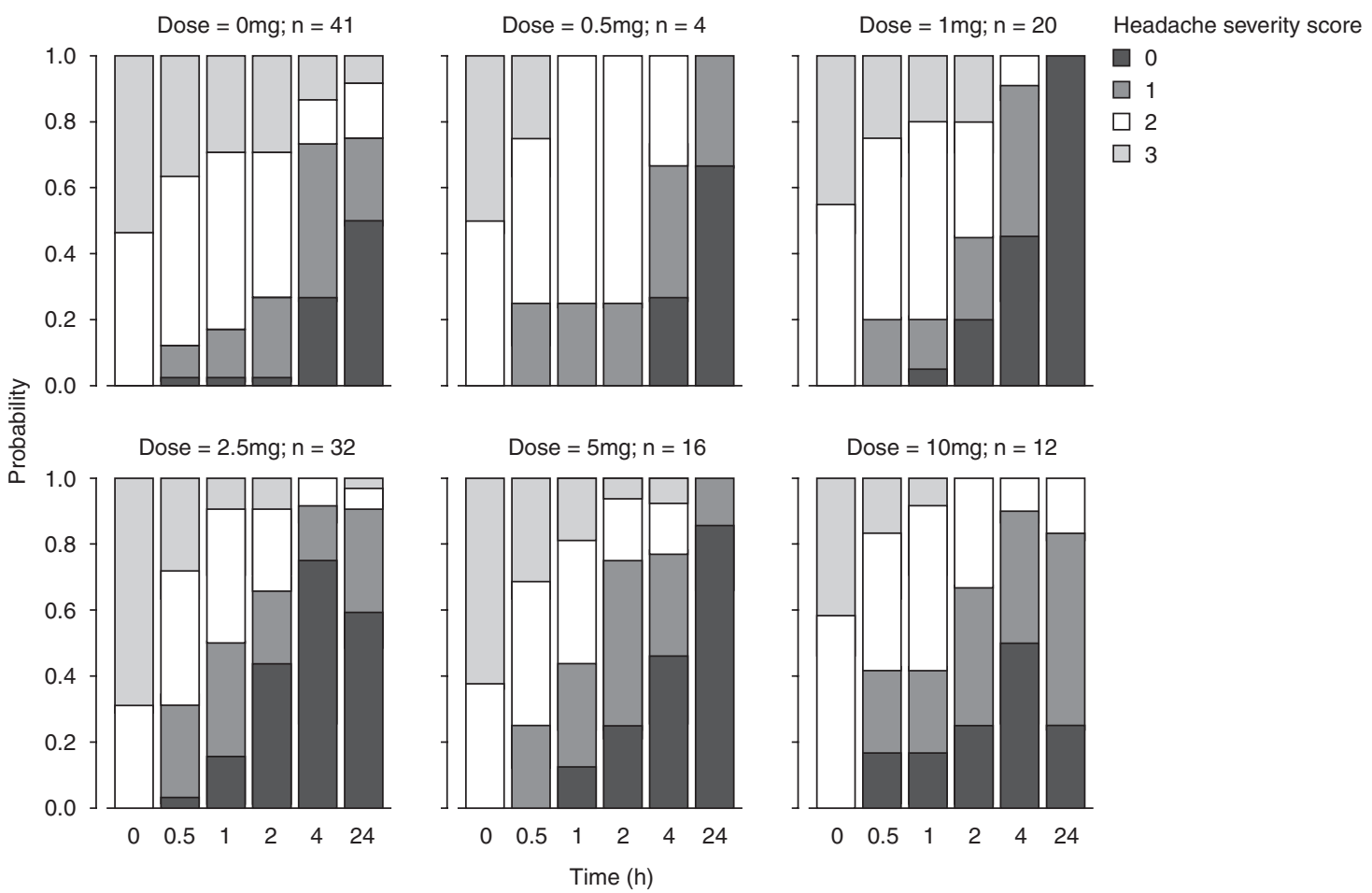

Fig. 1. Raw data probabilities of severity of headache as a function of time and dose. $\mathbf{n}=$ the number of subjects at the beginning of the treatment.

because of the nature of the pharmacodynamic response data (i.e. non-continuous and informative dropouts). ${ }^{[12]}$

\section{Methods}

\section{Patients and Study Design}

Information regarding patients and study design has been presented elsewhere. ${ }^{[9,11]}$ Briefly, 126 patients with a diagnosis of migraine (with or without aura) according to the criteria of the International Headache Society ${ }^{[13]}$ of at least 1 year were enrolled in this randomised, double-blind, placebo-controlled, multi-centre phase IIa study after giving written informed consent. The study was conducted in accordance with the Declaration of Helsinki. Other main inclusion criteria were a history of one to six migraine attacks per month for the preceding 6 months, an age at the onset of migraines of $\leq 50$ years, and a current age between 18 and 65 years.
Patients with significant medical (i.e. coronary artery disease by history, renal failure) or psychiatric illness were not allowed to participate in the study. A summary of demographic data including migraine history is given in table I.

After the first screening visit, patients were asked to return to the clinic when they had an acute moderate to severe migraine attack lasting no more than 6 hours. After randomisation, BIBN 4096 BS was given as a single intravenous 10 minute infusion at doses of $0.25,0.5,1,2.5,5$, or $10 \mathrm{mg}$ to $1,4,20,32$, 16 , and 12 patients, respectively. Placebo (xylitol) was also intravenously infused in 10 minutes to 41 patients. Dose selection was based on a group-sequential adaptive treatment-assignment design in order to identify the lowest dose of BIBN 4096 BS that is superior to placebo with a low number of patients and to minimise exposure of patients to non-efficacious doses. ${ }^{[14,15]}$ Groups of six patients were treated, with four patients receiving BIBN 

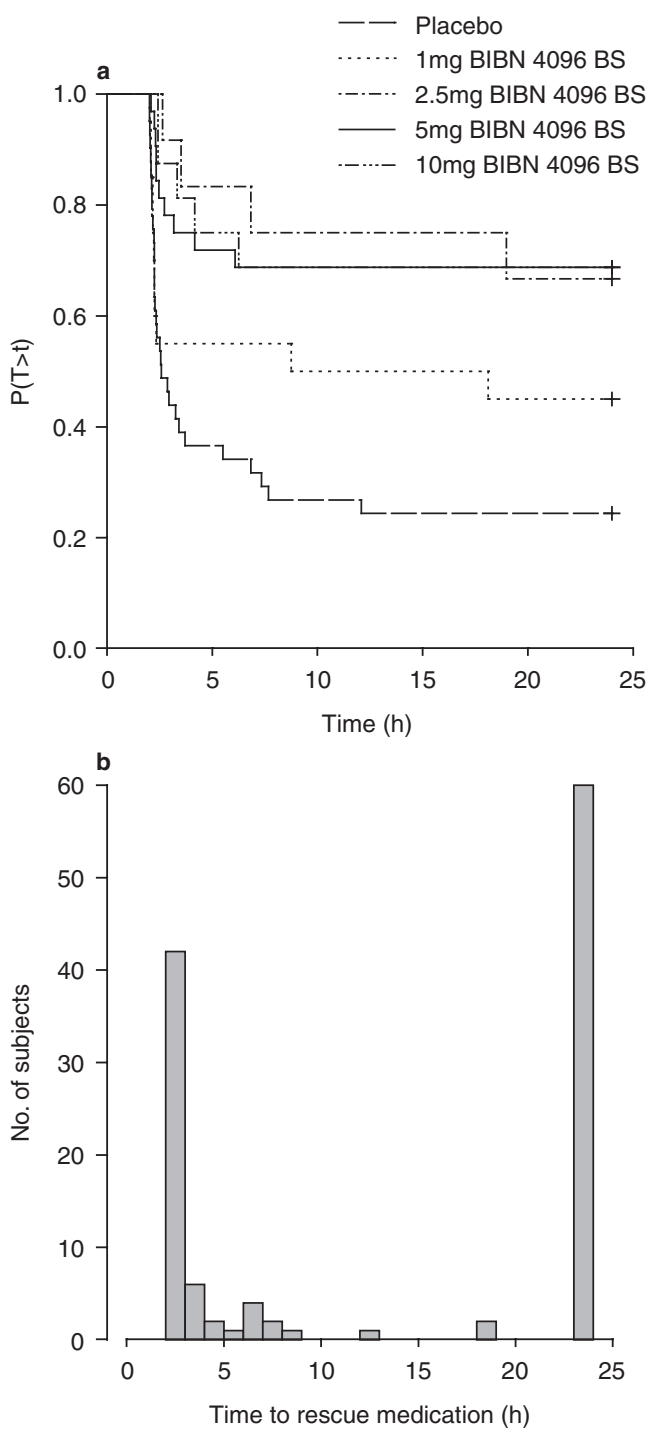

Fig. 2. (a) The Kaplan-Meier plot showing the fraction of the subjects receiving BIBN 4096 BS who remained in the study at least to time $t[P(T>t)]$. (b) The distribution of the times of rescue medication. The 24-hour bar corresponds to individuals that did not take rescue medication during the study period (censored data). $\mathbf{P}=$ probability; $\mathbf{t}=$ time; $\mathbf{T}=$ time after $\mathbf{t}$ when rescue medication was taken.

4096 BS and two patients receiving placebo. Starting with the dose of $1.0 \mathrm{mg}$, the dose for the next group was determined on the basis of the response observed in the preceding group. The dose in the next group was decreased if at least three of the four patients in the BIBN 4096 BS group had a response; otherwise, the dose was increased.

Migraine headache was evaluated by the investigator using the Headache Severity Scale $(3=$ severe; $2=$ moderate $;=$ mild $; 0=$ none) at baseline, $0.5,1$, 2, 4, and 24 hours after the start of the infusion. Patients remained in the clinic until at least 4 hours after the start of the infusion. The 24-hour measurement was recorded by telephone. Patients who did not show pain relief by 2 hours (reduction from headache severity scores of 3 or 2 to 1 or 0 ) after the start of the infusion were allowed to take rescue medication. Rescue medication was defined as any medication other than study drug, given to the patient for relief of headache pain or associated symptoms.

Blood samples $(8 \mathrm{~mL})$ were taken from a forearm vein on the opposite site of the infusion site predose, 10, 30 minutes, 1, 2, and 4 hours after the start of the infusion. Plasma was obtained from centrifugation and stored at $-20^{\circ} \mathrm{C}$ until analysis for BIBN 4096 BS concentrations with an accurate and sensitive analytical high-performance liquid chromatography method. ${ }^{[11]}$

\section{Pharmacodynamic Data}

A total of 641 headache severity scores were obtained during the study and used in the analysis. Figure 1 shows the distribution of the probabilities for each score by dosage group and measurement time. Severity of headache scores obtained at times $>2$ hours for those patients that took rescue medication were not included in the analysis. 504 measurements were recorded at times $\leq 2$ hours, and 76 and 61 observations were obtained from patients that did not ask for rescue medication at 4 hours and 24 hours after administration, respectively.

Figure 2 shows the Kaplan-Meier plot representing the probability of remaining in the study (i.e. patients that did not take rescue medication) as a function of the administered dose of BIBN 4096 BS and the distribution of times where rescue medication was taken. 


\section{Data Analysis}

Data from headache severity scores and time to rescue medication obtained from all the patients involved in the study were fitted simultaneously under the population approach using the Laplacian likelihood estimation method implemented in the nonlinear mixed-effect modelling software NONMEM version V. ${ }^{[16]}$ Headache severity scores were considered as ordered categorical variables and were fitted using logistic regression, and time to rescue medication was a time-to-event response variable described by survival analysis. ${ }^{[17,18]}$

Once a model providing an adequate description of the data without the incorporation of covariates was selected, patient characteristics listed in table I were explored for significance using the generalised additive model (GAM) approach implemented in the software Xpose version 3. ${ }^{[19,20]}$ The covariates initially selected during the GAM analysis are further tested for significance in NONMEM using the forward inclusion and backward elimination approach.

Model selection was done based on a number of criteria, such as the goodness-of-fit plots, precision of model parameter estimates, and the difference in the minimum value of the objective function $(-2$ $\log$ [likelihood]; -2LL) provided by NONMEM. A difference of 6.63 points in $-2 \mathrm{LL}$ between two nested models differing by one parameter was considered significant at the $1 \%$ level.

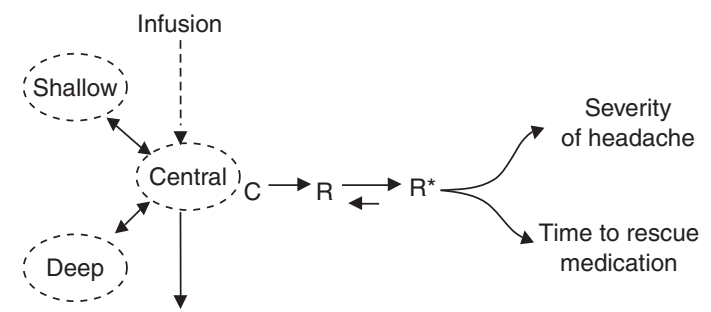

Fig. 3. Schematic representation of the selected pharmacokinetic and pharmacodynamic model. $\mathbf{C}=$ the model-predicted drug concentrations in plasma; $\mathbf{R}=$ the inactive form of the calcitonin generelated peptide (CGRP) receptor; $\mathbf{R}^{*}=$ the active form of the CGRP receptor, which has been related to the severity of headache and time to rescue medication using logistic regression and time-toevent analysis.
Results from the population pharmacokinetic analyses are presented as model parameter estimates together with their corresponding relative standard errors computed as the ratio between the standard error and the model parameter estimate.

\section{Drug-Receptor Interaction Model}

BIBN 4096 BS binds to the CGRP receptor blocking the binding of endogenous CGRP. Data from figure 1 show that BIBN $4096 \mathrm{BS}$ shows remaining effects at least 24 hours after administration, a result that is compatible with a slow dissociation of the drug-receptor complex, and which is supported from receptor binding studies performed in vitro. ${ }^{[7]}$ Figure 3 shows schematically the model used to relate the time course of the drug in plasma with severity of headache and time to rescue medication through the fraction of CGRP receptors that is blocked by BIBN 4096 BS ( $\left.R^{*}\right)$. Additionally, the effect compartment ${ }^{[21]}$ and the indirect response models were also fitted to the data, ${ }^{[22]}$ assuming that the lack of direct relationship between drug in plasma and response is caused by a distribution process between the plasma and biophase, or that the drug acts by stimulating or inhibiting the factors controlling the synthesis and degradation processes of the response.

Following the law of mass action the dynamics of $\mathrm{R}^{*}$ are represented by equation 1 :

$$
\frac{\mathrm{dR}^{*}}{\mathrm{dt}}=\mathrm{k}_{\mathrm{on}} \bullet \mathrm{C} \bullet \mathrm{R}-\mathrm{k}_{\mathrm{off}} \bullet \mathrm{R}^{*}
$$

where $\mathrm{dR}^{*} / \mathrm{dt}$ is the rate of change of $\mathrm{R}^{*}, \mathrm{C}$ corresponds to the predicted plasma concentrations of BIBN 4096 BS that were obtained from the pharmacokinetic model developed previously, ${ }^{[11]} \mathrm{R}$ is the concentration of unblocked receptors, and $\mathrm{k}_{\mathrm{on}}$ and $\mathrm{k}_{\text {off }}$ represent the second- and first-order rate constants representing the onset and offset of the anti-migraine effect elicited through the respective amount of CGRP receptors blocked by BIBN 4096 BS. Setting the total concentration of CGRP receptors $\left(\mathrm{R}_{\mathrm{T}}\right)$ arbitrarily to 1 , substituting $\mathrm{R}$ by $1-\mathrm{R}^{*}$, yields the expression (equation 2) used during model fitting: 

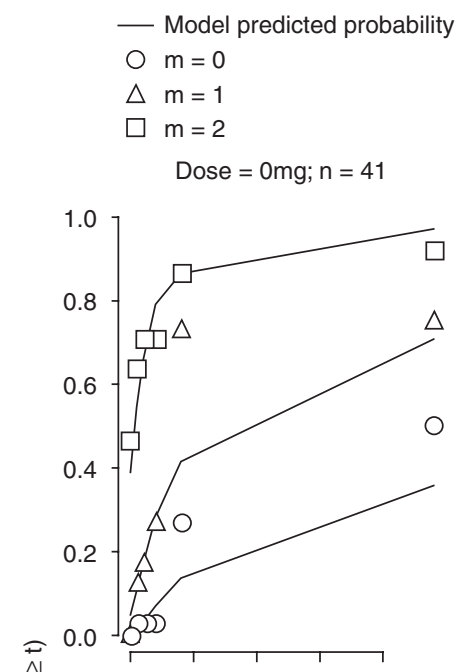

Dose $=0.5 \mathrm{mg} ; \mathrm{n}=4$

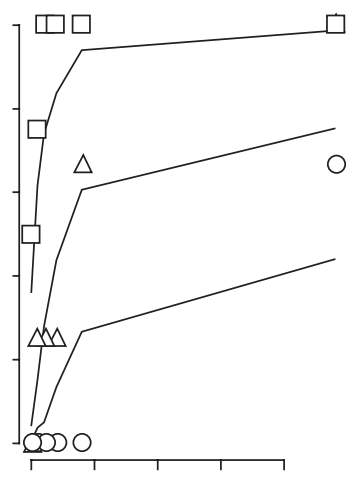

Dose $=5 \mathrm{mg} ; \mathrm{n}=16$

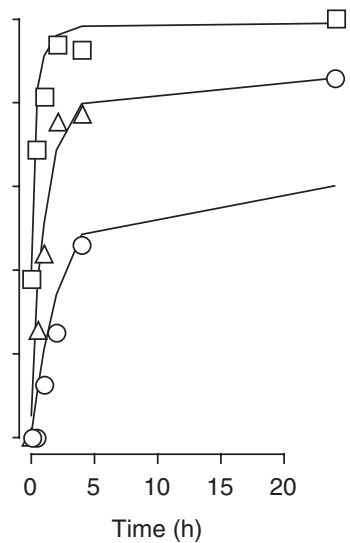

Dose $=1 \mathrm{mg} ; \mathrm{n}=20$

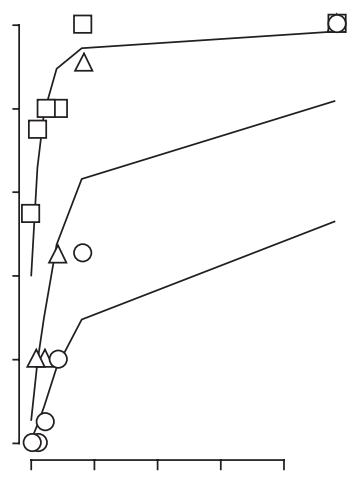

Dose $=10 \mathrm{mg} ; \mathrm{n}=12$

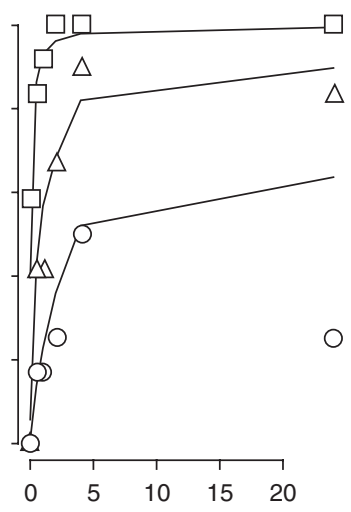

Fig. 4. Time profiles of the raw and model predicted probabilities showing that the severity of headache is lower or equal to $\mathrm{m}$ (cumulative probability), given that the subjects did not take rescue medication $[P(Y \leq m \mid T \geq t)] . n=$ the number of subjects at the beginning of the treatment; note that such number is decreased at times $>2$ hours because subjects took rescue medication. $\mathbf{P}=$ probability; $\mathbf{t}=$ time; $\mathbf{T}=$ time after $\mathrm{t}$ when rescue medication was taken; $\mathbf{Y}=$ observed score.

$$
\frac{\mathrm{dR}^{*}}{\mathrm{dt}}=\mathrm{k}_{\mathrm{on}} \bullet \mathrm{C} \bullet\left(1-\mathrm{R}^{*}\right)-\mathrm{k}_{\mathrm{off}} \bullet \mathrm{R}^{*}
$$

\section{Model for Severity of Headache}

In brief, the logistic regression model is represented by equation 3 :

$$
P Y_{i j}=\frac{e^{L}}{1+e^{L}}
$$

where $\mathrm{PY}_{\mathrm{ij}}$ represents the probability of getting a score $(\mathrm{Y}) \leq \mathrm{m}$ (cumulative probability) in the ith individual at the jth measurement time and $\mathrm{L}$ is the logit. An individual patient response was modeled as a conditional probability $\mathrm{P}\left(\mathrm{Y}_{\mathrm{ij}}=\mathrm{m} \mid \eta_{\mathrm{i}}\right)$ where $\eta_{\mathrm{i}}$ is the individual random effect. The set of individual $\eta$ values is assumed to be symmetrically distributed around 0 with variance $\omega^{2}$. The probability of hav-

(Eq. 3) ing score ' $m$ ' was coded as in equation 4: 


$$
\mathrm{P}\left(\mathrm{Y}_{\mathrm{ij}}=\mathrm{m} \mid \eta_{\mathrm{i}}\right)=\mathrm{P}\left(\mathrm{Y}_{\mathrm{ij}} \leq \mathrm{m} \mid \eta_{\mathrm{i}}\right)-\mathrm{P}\left(\mathrm{Y}_{\mathrm{ij}} \leq(\mathrm{m}-1) \mid \eta_{\mathrm{i}}\right)
$$

The logit (L) within equation 3 combines the contribution of baseline, placebo, and drug effects on the probability as follows (equation 5):

$$
\mathrm{L}=\mathrm{f}_{\text {baseline }}(\mathrm{m})+\mathrm{g}_{\text {placebo }}(\mathrm{t})+\mathrm{h}\left(\mathrm{R}^{*}\right)+\eta_{\mathrm{i}}
$$

where fbaseline $(\mathrm{m})$ describes the distribution of baseline scores and has the form (equation 6):

$$
\sum_{\mathrm{k}=1}^{\mathrm{m}} \beta_{\mathrm{k}}
$$

where $\beta_{\mathrm{k}}$ are the parameters defining the baseline probabilities. To account for a placebo effect ( $\mathrm{g}_{\text {placebo}}$ ), various time-dependent (maximum effect [Emax type, or biexponential) $)^{[18]}$ functions were tested. Drug effects were represented by a function (h) of the fraction of CGRP receptors that is blocked by BIBN 4096 BS. Four different models were tested for $\mathrm{h}$ : the linear, $\mathrm{E}_{\max }$, sigmoidal $\mathrm{E}_{\max }$, and the power model. The logistic model predicts an increased probability of showing mild or no pain when $\mathrm{R} *$ increases.

Goodness-of-fit graphs were created showing the mean raw data and mean model predicted cumulative probabilities for each dose group and time of measurement. The posterior predictive check was used to further explore the descriptive capability of the selected model. ${ }^{[23]}$ Severity of headache scores for 1000 datasets with the same characteristics of the original one were simulated using the selected model and its population model estimates. For the two dose groups with higher number of patients (placebo and 2.5mg) and for each of the simulated datasets $\mathrm{P}(\mathrm{Y} \leq 1)$ at 2 hours after the start of the administration of BIBN 4096 BS was computed and the distributions were represented graphically together with the corresponding raw data probability.

\section{Model for Rescue Medication}

The hazard function $[\mathrm{hz}(\mathrm{t})]$ in the analysis of the rescue medication data is defined as the instantaneous probability of taking rescue medication at a short period after $t(T)$, given that the patient has not taken rescue medication up to time $t$ and has the following general expression (equation 7): ${ }^{[24]}$

$$
h z(t)=\lim _{d t \rightarrow 0} \frac{P[t<T \leq t+d t \mid T>t]}{d t}
$$

where lim refers to limit.

The hazard function has to be nonnegative, but apart from that restriction $\mathrm{hz}(\mathrm{t})$ can adopt any shape which will be driven by the data. In the current analysis it has the form of equation 8 :

$$
\mathrm{hz}(\mathrm{t})=\mathrm{hz}_{0} \bullet\left(1-\frac{\mathrm{hz}_{\mathrm{max}} \bullet \mathrm{R}^{*}}{\mathrm{R}^{*}+\mathrm{hz}_{50}}\right) \cdot \mathrm{e}^{-\mathrm{k} \bullet \mathrm{t}}
$$

(Eq. 8)

where hzo, is the value of the hazard at baseline; $\mathrm{hz}_{\max }$ is the maximum decrease with respect to $\mathrm{hz}_{0}$ that $R^{*}$ can elicit $\left(0 \leq h z_{\max } \leq 1\right)$; hz50, is the value of $\mathrm{R}^{*}$ that produces half of maximal reduction in hzo; $\mathrm{k}$ is a parameter that allows to describe a time dependent decrease in hz. The model describes a decrease in $\mathrm{hz}(\mathrm{t})$ as $\mathrm{R}^{*}$ and $\mathrm{t}$ increase. Denoting $\mathrm{HZ}(\mathrm{t})$ as the cumulative $h z(t)$ value, the probability of remaining in the study $[\mathrm{P}(\mathrm{T}>\mathrm{t})]$ at least to time $\mathrm{t}$ is given by the survival function, $S(t)$, where $S(t)=e^{-H Z}$. Before rescue medication was allowed $S(t)$ was set to 1 , but the hazard was allowed to accumulate.

Goodness-of-fit plots include the nonparametric Kaplan-Meier plot created with the raw data superimposed by the model predicted probability of remaining in the study for each dose group.

\section{Simulations}

Model based computer simulations were performed with the aim to aid in the design and further development of CGRP receptor antagonists.

In the first case and taking into account that in principle an extravascular formulation represents a better choice than the intravenous administration, using the mean population estimates obtained from the selected model, $\mathrm{P}(\mathrm{Y} \leq 1)$ values at 2 hours after administration were simulated for several combinations of rate of absorption (represented by the firstorder rate constant of absorption $\left[\mathrm{k}_{\mathrm{a}}\right]$ ) and bioavai- 
lability values. $\mathrm{k}_{\mathrm{a}}$ values ranged from 0.34 to $6 \mathrm{~h}^{-1}$; values that correspond to times at which $90 \%$ of the administered dose have been absorbed within 6 and 0.5 hours, respectively. Bioavailability ranged from 0.05 to 1 .

For the second case $\mathrm{P}(\mathrm{Y} \leq 1)$ values at $2,4,6$, and 8 hours after the start of administration were simulated for several combinations of $\mathrm{k}_{\text {on }}$ and $\mathrm{k}_{\text {off. }}$ Values ranged between \pm 5 -fold of those estimated in the current analysis. In this simulation the pharmacokinetic behaviour of BIBN 4096 BS remained unchanged.

The results from the simulations were presented in tri-dimensional plots to facilitate the interpretation.

\section{Results}

The plasma concentration versus time profiles of BIBN 4096 BS could be best described by a three compartmental model. BIBN 4096 BS shows dose independent pharmacokinetic behaviour between the 0.25 and $10 \mathrm{mg}$ range, and none of the standard demographic characteristics elicited clinical relevant effects on the pharmacokinetic parameters. The mean population parameter estimates together with their degree of interpatient variability in brackets are: apparent volume of distribution (8.4 [53] L), total plasma clearance $(15.2$ [48] L/h), distribution clearance between the central and the shallow peripheral compartments $(15.5 \mathrm{~L} / \mathrm{h})$, apparent volume of the shallow peripheral compartment (4.4 [31] L), distribution clearance between the central and the deep peripheral compartments $(5.4[50] \mathrm{L} / \mathrm{h})$, and apparent volume of distribution of the deep peripheral compartment $(16.1 \mathrm{~L} / \mathrm{h}) .^{[11]}$

The effect compartment and the indirect response models performed worse than the receptor model described by equations 1 and 2 . The values of $-2 \mathrm{LL}$ corresponding the effect compartment, the indirect response, and the receptor models are 1592.774, 1609.785 , and 1579.774 , respectively.

Placebo effects were significant $(\mathrm{p}<0.01)$ and its time course was best described with an $\mathrm{E}_{\max }$ model using the time after drug administration as the predictor variable. The fraction of CGRP receptors blocked by BIBN 4096 BS was scaled linearly to describe drug effects on the severity of headache response. The $E_{\max }$, sigmoidal $E_{\max }$, and power models did not elicited a significant decrease in $-2 \mathrm{LL}$ ( $\mathrm{p}>0.05$ ), and also the drug effect parameters were estimated with very poor precision.

With regard to time to rescue medication, the model represented by equation 8 provided an estimate of h $z_{\max }$ equal to 1 and the resulted value of -2LL was almost identical to a model where the effects of $\mathrm{R}^{*}$ were described with an exponential model (equation 9):

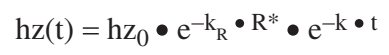

where $\mathrm{kR}_{\mathrm{R}}$ is the first-order rate constant describing an exponential decrease in $\mathrm{hz}_{0}$ as a function of $\mathrm{R}^{*}$.

Since this last model is simpler, it was the model finally selected. The term $\mathrm{e}^{-\mathrm{k} \bullet \mathrm{t}}$, represents a decrease in the hazard with time and its incorporation in the model improved the fit significantly $(\mathrm{p}<$ 0.001).

Figure 4 and figure 5 show that the selected pharmacodynamic model was capable to describe very well both types of observations (severity of headache and time to rescue medication). None of the covariates explored showed any statistical sig-

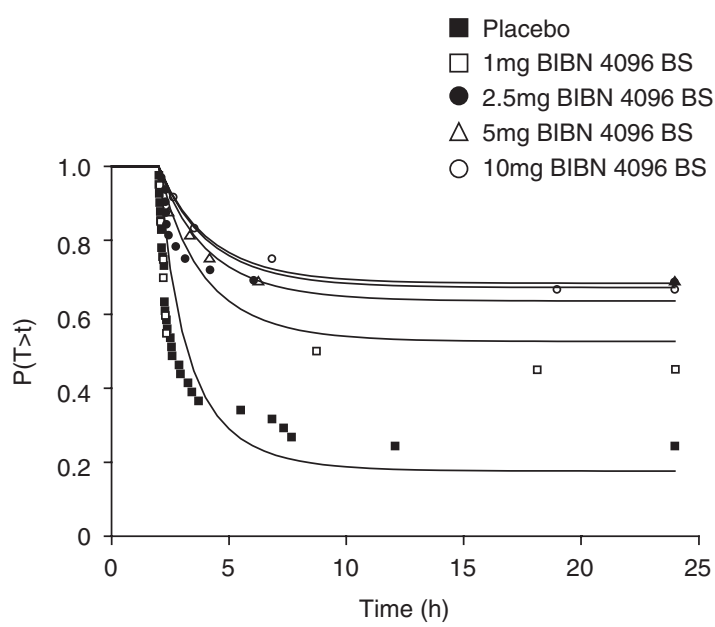

Fig. 5. Raw (symbols) and model-predicted (lines) fraction of subjects remaining in the study at least to time $t[P(T>t)]$ vs time and dose. $\mathbf{P}=$ probability; $\mathbf{t}=$ time; $\mathbf{T}=$ time after $\mathrm{t}$ when rescue medication was taken. 


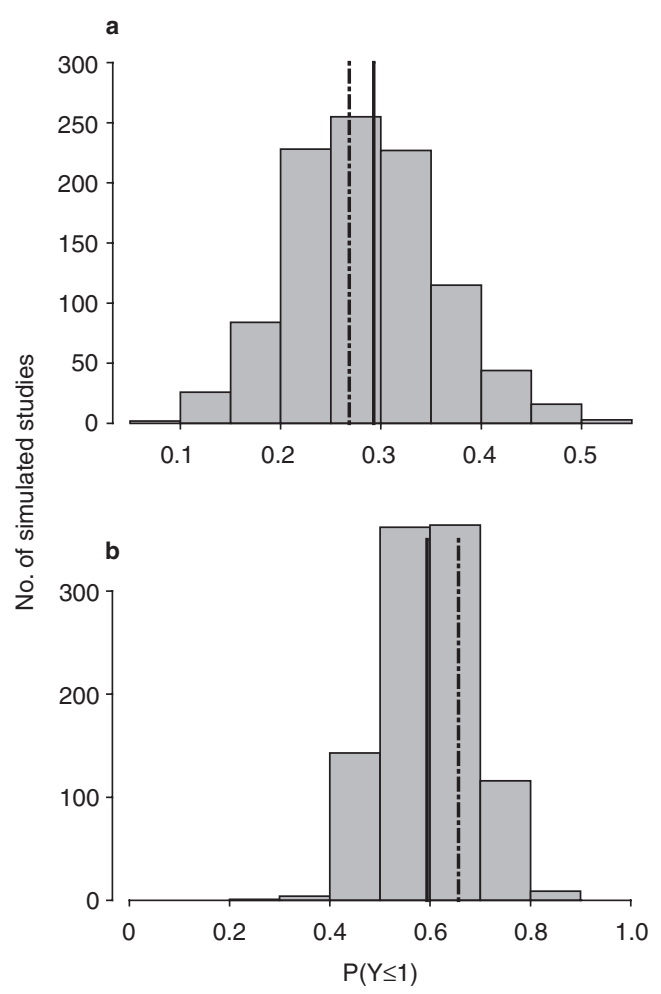

Fig. 6. Results from model validation using the posterior predictive check. Histograms represent the distribution of the mean $P(Y \leq 1)$ values obtained from 1000 simulated datasets in the placebo (a) and $2.5 \mathrm{mg}$ dose (b) groups. Vertical lines show the mean $P(Y \leq 1)$ values computed from the raw data (dashed) and from the simulations (solid lines).

nificance during the GAM approach. The results from the validation procedure shown in figure 6 confirm the model was supported by the data since there is a very good agreement between the model based simulated and observed $\mathrm{P}(\mathrm{Y} \leq 1)$ values. The mean $\mathrm{P}(\mathrm{Y} \leq 1)$ at 2 hours corresponding to the 1000 simulations are 0.29 and 0.6 for the 0 and $2.5 \mathrm{mg}$ dose groups, respectively, values that are very close to ones obtained from the observed data $(0.27$ and 0.65 , respectively). Parameter estimates of the pharmacodynamic model are listed in table II. All parameters were estimated with acceptable precision.

Figure 7 allows the exploration of the complete pharmacokinetic/pharmacodynamic model in detail, simulating the pharmacokinetics and pharmacodynamics of the main components of the model assum- ing placebo administration, or a 10-minute intravenous infusion of BIBN 4096 BS $2.5 \mathrm{mg}$.

Figures $7 \mathrm{a}$ and $7 \mathrm{~b}$ reflect the behaviour of the pharmacokinetic and the drug receptor binding models. The fraction of maximum receptor blockade corresponding to that dose, route and mode of administration was 0.8 , and occurred with a 2.5 -hour delay with respect to the $\mathrm{C}_{\max }$. Deblocking of receptors was slow compared with the decrease of BIBN 4096 BS in plasma and is controlled by the estimated value of $\mathrm{k}_{\text {off. }}$. With BIBN 4096 BS, it occurred with a half-life $\left(0.693 / \mathrm{k}_{\text {off }}\right)$ of 21 hours.

Figures $7 \mathrm{c}$ and $7 \mathrm{~d}$ show the pharmacodynamics predicted by the severity of headache (logistic) model. Figure 7c shows the change in the Logit from baseline induced by $\mathrm{R}^{*}$ (linearly scaled by parameter $\left.\theta_{\mathrm{SCL}}\right)$ and placebo. Figure $7 \mathrm{~d}$ represents the translation of the Logits into probabilities and shows the contribution of blocked receptors and placebo to

Table II. Model parameter estimates

\begin{tabular}{lll}
\hline Model parameters & Estimate & $\begin{array}{l}\text { Relative standard } \\
\text { errors }\end{array}$ \\
\hline $\begin{array}{l}\text { Receptor binding model } \\
\text { kon }(\mathrm{mL} / \mathrm{ng} / \mathrm{h})\end{array}$ & $1.5 \times 10^{-2}$ & 0.42 \\
$\mathrm{k}_{\mathrm{off}}\left(\mathrm{h}^{-1}\right)$ & $3.3 \times 10^{-2}$ & 0.46 \\
Severity of headache mode & & \\
$\beta_{1}$ & -0.81 & 0.36 \\
$\beta_{2}$ & -4.63 & 0.08 \\
$\beta_{3}$ & -7.17 & 0.09 \\
$\mathrm{PLmax}$ & 7.02 & 0.11 \\
$\mathrm{PL} 50(\mathrm{~h})$ & 2.62 & 0.22 \\
$\theta$ SCL & 3.01 & 0.24 \\
$\omega^{2}$ & 5.18 & 0.22 \\
Rescue medication model $^{2}$ & & \\
$\mathrm{hz}_{0}\left(\mathrm{~h}^{-1}\right)$ & 1.66 & 0.43 \\
$\mathrm{k}_{\mathrm{R}}\left(\mathrm{h}^{-1}\right)$ & 1.7 & 0.23 \\
$\mathrm{k}\left(\mathrm{h}^{-1}\right)$ & 0.42 & 0.22 \\
\hline
\end{tabular}

$\beta \boldsymbol{x}=$ set of baseline parameters of severity of headache; $\theta \mathrm{SCL}=$ parameter scaling the fraction of calcitonin gene-related peptide receptors blocked by BIBN $4096 \mathrm{BS}\left(\mathrm{R}^{\star}\right) ; \omega^{2}=$ population variance; $\mathbf{h z}_{0}=$ hazard value at baseline; $\mathbf{k}=$ first-order rate constant describing an exponential decrease in hzo as a function of time after the start of administration; $\mathbf{k}_{\text {on }}=$ second-order rate constant of activation of the anti-migraine effect; $\mathbf{k}_{\mathrm{off}}=$ first-order rate constant of inactivation of the anti-migraine effect; $\mathbf{k}_{\mathbf{R}}=$ first-order rate constant describing an exponential decrease in hzo as a function of $\mathrm{R}^{*} ; \mathrm{PL}_{50}=$ time at which the placebo effect is half of maximum; $\mathrm{PL}_{\max }=$ maximum placebo effect. 

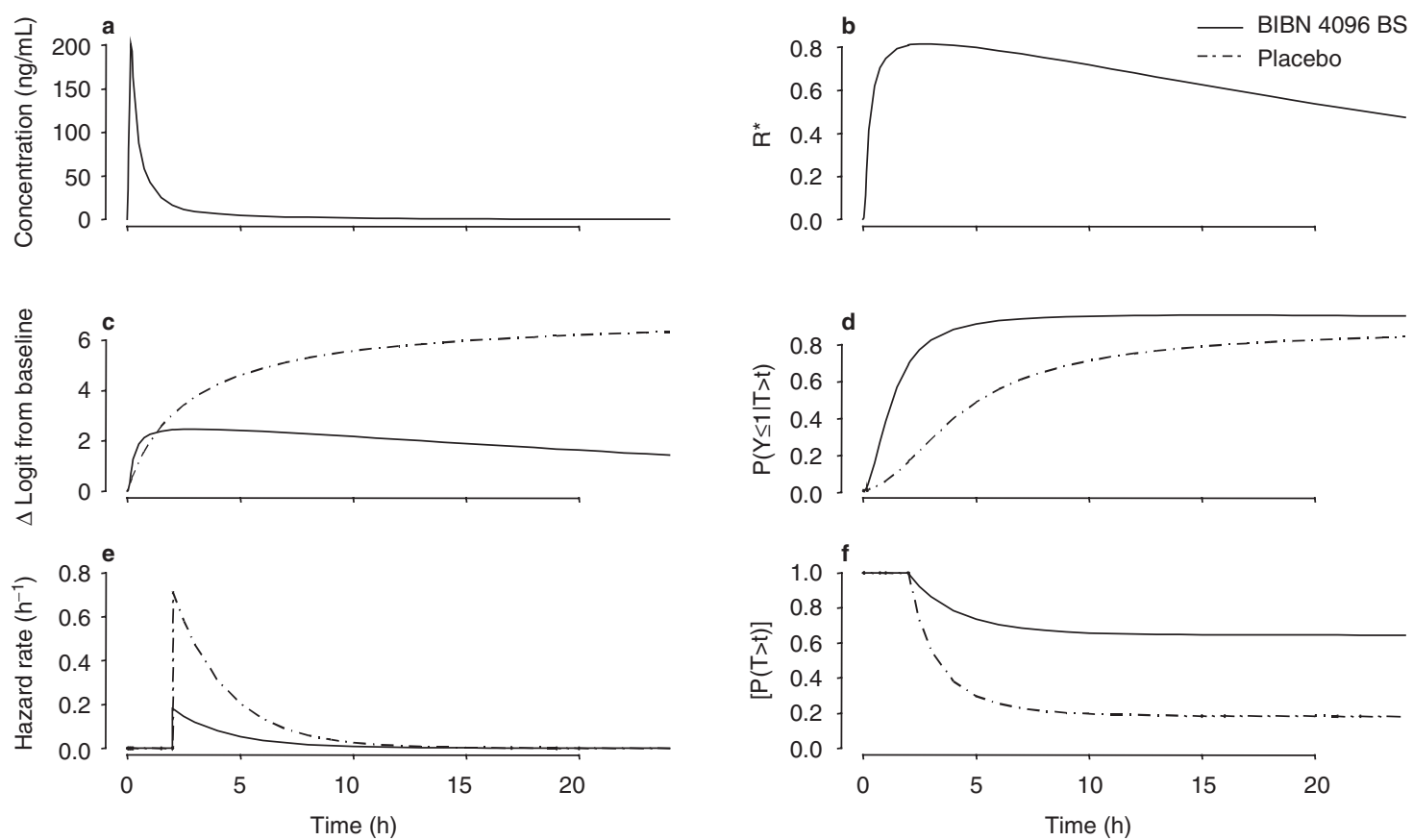

Fig. 7. Graphical exploration of the different components of the pharmacokinetic/pharmacodynamic model assuming a 10-minute intravenous infusion of BIBN 4096 BS 2.5mg. (a) Plasma concentration vs time profile. (b) Active form of blocked calcitonin gene-related peptide receptor $\left(R^{\star}\right)$ vs time profile. (c) Absolute change in the Logit elicited by placebo and by BIBN 4096 BS vs time profiles. (d) Time profiles of $P(Y \leq 1)$ corresponding to the placebo and drug-treated groups. (e) Hazard rate as a function of time in the placebo and drug-treated groups. (f) Fraction of the subjects remaining in the study at least to time $t[P(T>t)$, survival curve], in the placebo and drug-treated groups. $\mathbf{P}=$ probability; $\mathbf{t}=$ time; $\mathbf{T}=$ time after $\mathbf{t}$ when rescue medication was taken; $\mathbf{Y}=$ observed score.

the cumulative probability of achieving pain relief. As expected, the major impact of the effects is induced by the drug blocking the receptor, especially at shorter times after drug administration.

Figures $7 \mathrm{~d}$ and $7 \mathrm{e}$ correspond to the rescue medication model. At the time the rescue medication was allowed, the value of the hazard decreased from 0.75 to 0.2 in the simulated drug-treated group compared with placebo, due to the degree of the receptor blockade. The decrease with time in the hazard seen in the placebo group reflects the time effects quantified by $\mathrm{e}^{-\mathrm{k} \bullet \mathrm{t}}$.

\section{Discussion}

This report presents the application of population pharmacokinetic/pharmacodynamic modeling for BIBN $4096 \mathrm{BS}$, the first compound of the new class of CGRP receptor antagonists. Since the ultimate goal of this analysis is to assist in the development of CGRP receptor antagonists by simulation technology, the developed model should allow discriminating between pharmacokinetics and pharmacodynamics, and within pharmacodynamics, those properties that are related to the drug and those belonging to the system. The model applied in the current study fulfills those requirements. Pharmacokinetics were described with a standard three compartment model with first-order elimination and can be clearly differentiated from the time course of drug action. ${ }^{[11]}$ With regard to pharmacodynamics, drug properties are represented by the receptor binding model. The system related properties are represented by the baseline, placebo, and the parameters scaling $\mathrm{R}^{*}$. Nevertheless, taking into account the absence of information at the receptor/biophase level, and the mechanistic models that have been developed over the last years, the model presented here 
can be classified in the category of semi-mechanistic models. ${ }^{[25]}$

The time course of pain response during drugtreated migraine attacks has been described before by using the population pharmacokinetic/pharmacodynamic approach. ${ }^{[26]}$ Generally, time profiles similar to those found after administration of BIBN 4096 BS were also found for triptans and they were modeled using the effect compartment model. ${ }^{[21]}$ Delays between drug kinetics in plasma and response can be described with different models, which differ in the location of the rate-limiting step within the cascade of events controlling the course of the response. ${ }^{[27]}$ Results from our analysis suggest that the rate-limiting step is located at the receptor level and consists on a slow rate of receptor inactivation. This finding is supported by data obtained in an in vitro binding assay where a slow dissociation rate constant with a value of $0.0018 \mathrm{~min}^{-1}$ was obtained after binding of $\left[{ }^{3} \mathrm{H}\right] \mathrm{BIBN} 4096 \mathrm{BS}$ to human neuroblastoma SK-N-MC cell membranes expressing functional CGRP receptors. ${ }^{[7]}$ Two decades ago, during the modeling of the time course of the effects of the anti-migraine agent ergotamine, a slow rate of receptor inactivation (seen also in vitro) was also suggested as a possibility to explain the delay between kinetics in plasma and response. ${ }^{[28]}$ Two components contribute to pain relief in the model developed (placebo and drug effects), and only one to pain severity (CGRP). It is also possible that timedependent mechanisms such as development of central sensitisation play a significant role. However, due to lack of data the description of their kinetics and significance is not possible.

The data presented here include ordered categorical and survival data. Models describing the time course of headache severity scores and fitting the time to rescue medication as a function of the observable headache severity scores and time have been published in the past. ${ }^{[17,18]}$ More recently, a joint model has been proposed to deal with informative dropouts by describing the survival data as a function of the underlying unobserved data. ${ }^{[29]}$ In the current analysis, time to rescue medication was not described as a function of the observable severity of headache, or vice versa. Here, both types of response data were fitted simultaneously by using the receptor binding model. Having a model describing the probability of severity of headache is very useful, because it allows to compute $[\mathrm{P}(\mathrm{Y} \leq 1)]$, reflecting the response rate at 2 hours $\left([\mathrm{P}(\mathrm{Y} \leq 1)]_{\mathrm{t}}=2 \mathrm{~h}\right)$ after administration, an important efficacy endpoint for the anti-migraine agents. The model for time to rescue medication is also interesting since the concept of time-to-event analysis is receiving increasing attention in migraine research. ${ }^{[30]}$

Comparing model estimates at baseline for severity of headache and time to rescue medication is difficult; however, comparisons can be made for placebo and time effects and for the scaling parameters of the $\mathrm{R}^{*}$ effects. For example, the estimate of PL50 of 2.6 hours indicates the time after administration at which the placebo effect is half of maximum; this finding is similar to the half-life estimate derived from the $\mathrm{k}$ parameter governing the time effects in the hazard function $[(0.693 / 0.4)=1.73$ hours]. In the case of $\mathrm{R}^{*}$ effects and for a fraction of blocked CGRP receptors of 0.5 , the induced abso-

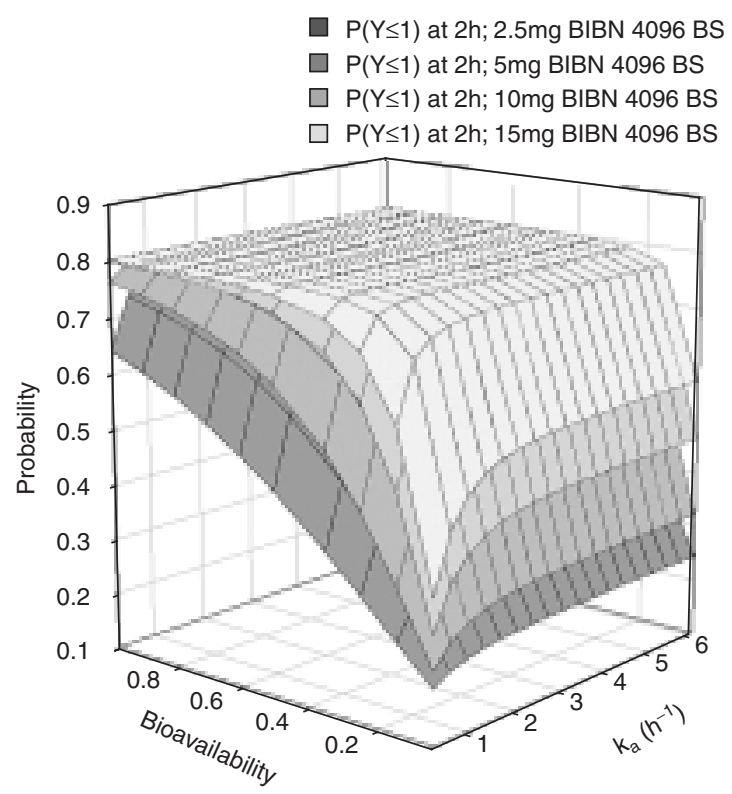

Fig. 8. Model based simulated values of $P(Y \leq 1)$ at 2 hours after administration as a function of bioavailability, first-order rate constant of absorption $\left(\mathrm{k}_{\mathrm{a}}\right)$ and administered dose. 

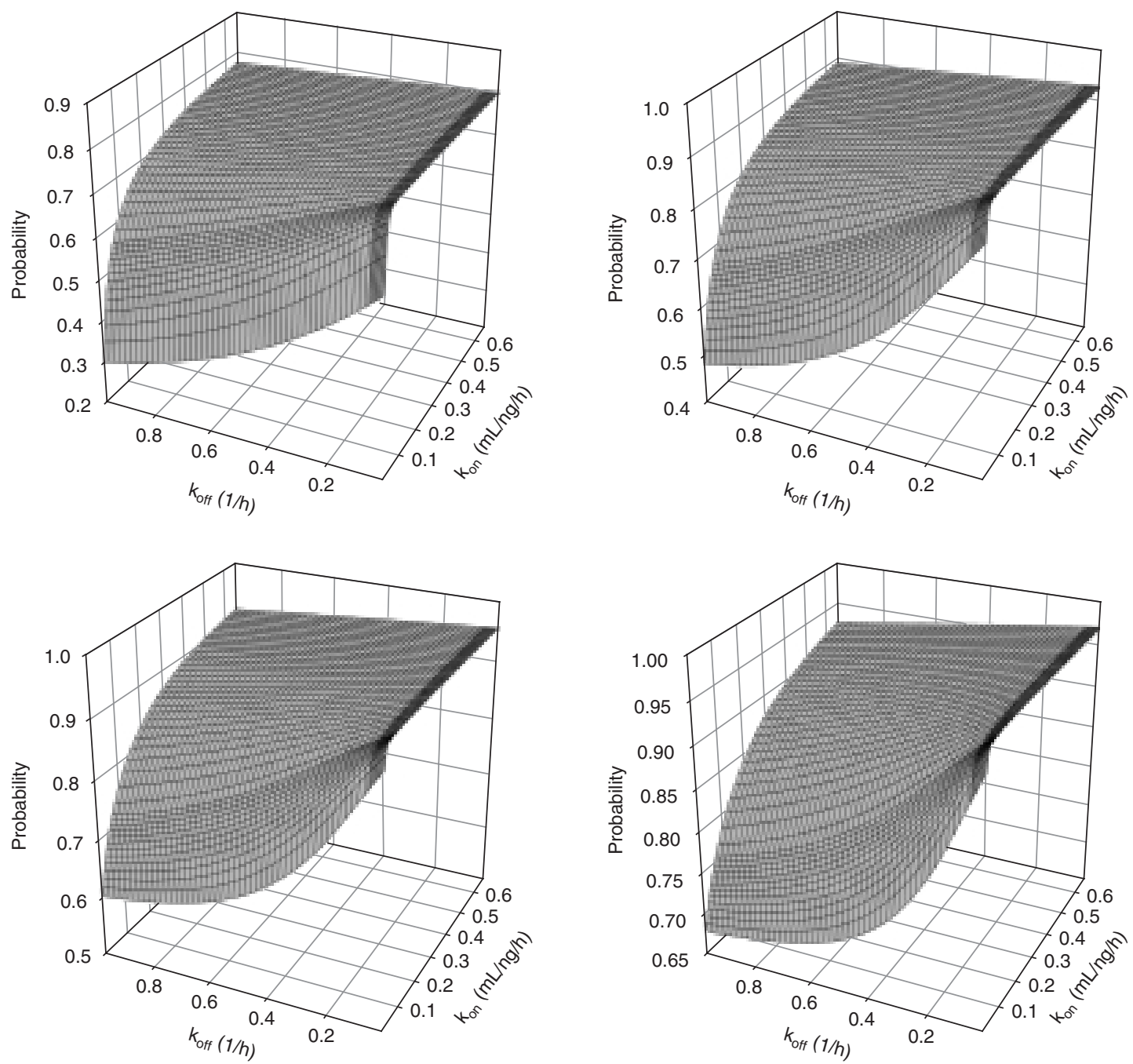

Fig. 9. Model based simulated values of $P(Y \leq 1)$ at 2, 4, 6 and 8 hours after administration of a 10-minute intravenous infusion of $B I B N 4096$ BS $2.5 \mathrm{mg}$ as a function of the second- and first-order rate constants of activation (kon) and inactivation (koff) of the anti-migraine effect.

lute changes in the value of the Logit and hazard function were 1.5 and 0.95 , respectively.

Ideally, as an anti-migraine medication should be given via routes other than intravenous, the model can be used to explore the influence of the absorption pharmacokinetics on the clinical outcome. Figure 8 shows the results of the simulated $[\mathrm{P}(\mathrm{Y} \leq 1)]_{\mathrm{t}}=$ $2 \mathrm{~h}$, based on different combinations of $\mathrm{k}_{\mathrm{a}}$ and bioavailability values in case BIBN 4096 BS would be given by an extravascular administration and assuming that the disposition is not affected. For bioavailabilities $<0.5$ a rapid absorption is required $\left(\mathrm{k}_{\mathrm{a}} \sim 1-2 \mathrm{~h}^{-1}\right)$ to achieve the maximum possible response rate for a particular dose, otherwise the $\mathrm{k}_{\mathrm{a}}$ has little impact. In contrast, the bioavailability is important to obtain the desired response rate value. Assuming that the total amount of drug in a particular dosage form can be, for example, $15 \mathrm{mg}$, it ap- 
pears that a bioavailability between 0.2 and 0.4 should at least be achieved to reach a response rate of 0.6 at 2 hours. When applying those results to potential oral formulations, attention should be paid to the fact that migraine attacks might alter drug absorption increasing the variability in the pharmacokinetic profiles and consequently affecting the clinical response. ${ }^{[31]}$ Simulations can be performed increasing the degree of intersubject variability, and using more complex absorption models than the one shown here for illustration purposes. $^{[32,33]}$

Figure 9 shows the results from simulations where $[\mathrm{P}(\mathrm{Y} \leq 1)]_{\mathrm{t}}=2-8 \mathrm{~h}$ values were plotted as a function of different combinations of $\mathrm{k}_{\mathrm{on}}$ and $\mathrm{k}_{\text {off }}$ assuming no change in the pharmacokinetics. A value of $\mathrm{k}_{\text {on }}$ of at least $0.1 \mathrm{~mL} / \mathrm{ng} / \mathrm{h}$ is required to have a $[\mathrm{P}(\mathrm{Y} \leq 1)]_{\mathrm{t}}=2 \mathrm{~h}$ of 0.6 given that $\mathrm{k}_{\text {off }}$ is $<0.53$ $\mathrm{h}^{-1}$. To achieve a $[\mathrm{P}(\mathrm{Y} \leq 1)]_{\mathrm{t}}=2 \mathrm{~h}$ of 0.6 , values of $\mathrm{k}_{\text {on }}$ and $\mathrm{k}_{\text {off }}$ should be $>0.044 \mathrm{~mL} / \mathrm{ng} / \mathrm{h}$ and $<0.23 \mathrm{~h}^{-1}$, respectively. At later times, after administration, higher values of $\mathrm{k}_{\text {off }}$ are associated with faster offset of the response. We realise that the estimates of those two parameters can be very different from those to be calculated from in vitro, for many reasons, such as experimental conditions, and the fact that the receptor binding model proposed in the current manuscript involves probably distribution kinetics to the effect site and signal transduction events. Therefore, the results of that simulation should be explored searching for general patterns instead of concrete values of the two first-order rate constants.

\section{Conclusion}

A population pharmacokinetic/pharmacodynamic model of BIBN 4096 BS, the first compound of the new class of CGRP receptor antagonists, has been developed for anti-migraine effects characterised by severity of headache and time to rescue medication. The model is semi-mechanistic and it was validated properly. Simulations exploring the effect of important parameters such as rate of absorption, bioavailability, and the rate of receptor activation and inactivation were performed. The rate of absorption seems to play a minor role; however, at least bioavailability fractions of $0.2-0.3$ should be obtained for extravascular dosage formulations. With regard to the receptor binding characteristics of new follow-up compounds, simulation shows that molecules with fast $\mathrm{k}_{\text {on }}$ and low $\mathrm{k}_{\text {off }}$ are the most promising.

\section{Acknowledgements}

The work was supported by Boehringer Ingelheim Pharma GmbH \& Co. KG; Biberach, Germany. Dr Iñaki F. Trocóniz has received research funding from Boehringer Ingelheim Pharma GmbH \& Co. KG. Dr Jan-Markus Wolters, Christiane Tillmann, Dr Hans Guenter Schaefer, and Dr Willy Roth are employees of Boehringer Ingelheim Pharma GmbH \& Co. KG.

\section{References}

1. Lipton RB, Stewart WF, Diamond S, et al. Prevalence and burden of migraine in the United States: data from the American Migraine Study II. Headache 2001 Jul-Aug; 41 (7): 646-57

2. Gallai V, Sarchielli P, Floridi A, et al. Vasoactive, peptide levels in the plasma of young migraine patients with or without aura assessed both interictally and ictally. Cephalalgia 1995 Oct; 15 (5): 384-96

3. Jansen-Olesen I, Mortensen A, Edvinsson L. Calcitonin generelated peptide is released from capsaicin-sensitive nerve fibres and induces vasodilatation of human cerebral arteries concomitant with activation of adenylyl cyclase. Cephalalgia 1996 Aug; 16 (5): 310-6

4. Durham PL. CGRP-receptor antagonists: a fresh approach to migraine therapy? N Engl J Med 2004 Mar; 350 (11): 1073-5

5. Buzzi MG, Carter WB, Shimizu T, et al. Dihydroergotamine and sumatriptan attenuate levels of CGRP in plasma in rat superior sagital sinus during electrical stimulation of the trigeminal ganglion. Neuropharmacology 1991 Nov; 30 (11): 1193-200

6. de Hoon JN, Willigers JM, Troost J, et al. Vascular effects of 5-HT1B/1D-receptor agonists in patients with migraine headaches. Clin Pharmacol Ther 2000 Oct; 68 (4): 418-26

7. Schindler M, Doods HN. Binding properties of the novel, nonpeptide CGRP receptor anatgonist radioligand, [(3)H] BIBN 4096 BS]. Eur J Pharmacol 2002 May; 442 (3): 187-93

8. Doods H. Development of CGRP antagonists for the treatment of migraine. Curr Opin Investig Drugs 2001 Sep; 2 (9): 1261-8

9. Olesen J, Diener HC, Husstedt IW, et al. Calcitonin gene-related peptide receptor antagonist BIBN 4096 BS for the acute treatment of migraine. N Engl J Med 2004 Mar; 350 (11): 1104-10

10. Iovino M, Feifel U, Yong CL, et al. Safety, tolerability and pharmacokinetics of BIBN 4096 BS, the first selective small molecule calcitonin gene-related peptide receptor antagonist, following single intravenous administration in healthy volunteers. Cephalalgia. 2004 Aug; 24 (8): 645-56

11. Trocóniz IF, Wolters JM, Schaefer HG, et al. Population pharmacokinetic modelling of BIBN 4096 BS, the first compound of the new class of calcitonin gene-related peptide receptor antagonists. Eur J Pharm Sci 2004 Jul; 22 (4): 287-95 
12. Sheiner LB, Steimer JL. Pharmacokinetic/pharmacodynamic modeling in drug development. Annu Rev Pharmacol Toxicol 2000; 40: 67-95

13. Headache Classification Committee of the International Headache Society. Classification and diagnostic criteria for headache disorders, cranial neuralgias and facial pain. Cephalalgia 1988; 8 Suppl. 7: 1S-96S

14. Moller S. An extension of the continual reassessment methods using a preliminary up-and-down design in a dose finding study in cancer patients, in order to investigate a greater range of doses. Stat Med 1995 May; 14 (9-10): 911-22

15. Roon KI, Olesen J, Diener HC, et al. No acute antimigraine efficacy of CP-122,288, a highly potent inhibitor of neurogenic inflammation: results of two randomized, double-blind, placebo-controlled clinical trials. Ann Neurol 2000 Feb; 47 (2): 238-41

16. Beal SL, Sheiner LB, editors. NONMEM users' guides. San Francisco (CA): NONMEM Project Group, University of California at San Francisco, 1992

17. Sheiner LB. A new approach to the analysis of the analgesic drug trials, illustrated with bromfenac data. Clin Pharmacol Ther 1994 Sep; 56 (3): 309-22

18. Mandema JW, Stanski DR. Population pharmacodynamic model for ketorolac analgesia. Clin Pharmacol Ther 1996 Dec; 60 (6): 619-35

19. Mandema JW, Verotta D, Sheiner LB. Building population pharmacokinetic-pharmacodynamic models: I. Models for covariate effects. J Pharmacokinet Biopharm 1992 Oct; 20 (5): 511-28

20. Jonsson EN, Karlsson MO. Xpose: an Splus based population pharmacokinetic/pharmacodynamic model building aid for NONMEM. Comput Methods Programs Biomed 1999 Jan; 58 (1): 51-64

21. Sheiner LB, Stanski DR, Vozeh S, et al. Simultaneous modeling of pharmacokinetics and pharmacodynamics: application to dtubocurarine. Clin Pharmacol Ther 1979 Mar; 25 (3): 358-71

22. Dayneka NL, Garg V, Jusko WJ. Comparison of four basic models of indirect pharmacodynamic responses. J Pharmacokinet Biopharm 1993 Aug; 21 (4): 457-78

23. Yano Y, Beal SL, Sheiner LB. Evaluating pharmacokinetic/ pharmacodynamic models using the posterior predictive check. J Pharmacokinet Pharmacodyn 2001 Apr; 28 (2): 171-92
24. Kalbfleish JD, Prentice RL. The statistical analysis of failure time data. New York: John Wiley, 1980

25. Visser SA, Huntjens DRH, Van der Graff PH, et al. Mechanismbased modeling of the pharmacodynamic intercation of alphaxalone and midazolam in rats. J Pharm Exp Ther 2003 Nov; 307 (2): 765-75

26. Nestorov I, Graham G, Duffull S, et al. Modeling and stimulation for clinical trial design involving a categorical response: a phase II case study with naratriptan. Pharm Res 2001 Aug; 18 (8): 1210-9

27. Jusko WJ, Ko HC, Ebling WF. Convergence of direct and indirect pharmacodynamic response models. J Pharmacokinet Biopharm 1995 Feb; 23 (1): 5-8

28. Tfelt-Hansen P, Paalzow L. Intramuscular ergotamine: plasma levels and dynamic activity. Clin Pharmacol Ther 1985 Jan; 37 (1): 29-35

29. $\mathrm{Hu} \mathrm{C}$, Sale ME. A joint model for nonlinear longitudinal data with informative dropout. J Pharmacokinet Pharmacodyn 2003 Feb; 30 (1): 83-103

30. Allen C, Jiang K, Malbecq W, et al. Time-to-event analysis, or who gets better sooner? An emerging concept in headache study methodology. Cephalagia 1999 Jul; 19 (6): 552-6

31. Boyle R, Behan PO, Sutton JA. A correlation between severity of migraine and delayed gastric emptying measured by an epigastric impedance method. Br J Clin Pharmacol 1990 Sep; 30 (3): 405-9

32. Troconiz IF, Armenteros S, Planelles MV, et al. Pharmacokinetic-Pharmacodynamic Modelling of the antipyretic effect of two oral formulations of ibuprofen. Clin Pharmacokinet 2000 Jun; 38 (6): 505-18

33. Lindberg-Freijs A, Karlsson MO. Dose dependent absorption and linear disposition of cyclosporin A in rat. Biopharm Drug Dispos 1994 Jan; 15 (1): 75-86

Correspondence and offprints: Dr Jan-Markus Wolters, Department of Project Management R\&D, Boehringer Ingelheim Pharma GmbH \& Co. KG, Birkendorfer Strasse 65, Biberach, 88397, Germany.

E-mail: markus.wolters@bc.boehringer-ingelheim.com 